\title{
In vitro study of combined cilengitide and radiation treatment in breast cancer cell lines
}

Tim Lautenschlaeger ${ }^{1,2^{*}+}$, James Perry ${ }^{1,2+}$, David Peereboom ${ }^{3}$, Bin Li ${ }^{1,2}$, Ahmed Ibrahim ${ }^{1,2,4}$, Alexander Huebner ${ }^{1,2}$, Wei Meng ${ }^{1,2}$, Julia White ${ }^{1}$ and Arnab Chakravarti ${ }^{1,2}$

\begin{abstract}
Background: Brain metastasis from breast cancer poses a major clinical challenge. Integrins play a role in regulating adhesion, growth, motility, and survival, and have been shown to be critical for metastatic growth in the brain in preclinical models. Cilengitide, an av $\beta 3 / a v \beta 5$ integrin inhibitor, has previously been studied as an anti-cancer drug in various tumor types. Previous studies have shown additive effects of cilengitide and radiation in lung cancer and glioblastoma cell lines. The ability of cilengitide to enhance the effects of radiation was examined preclinically in the setting of breast cancer to assess its possible efficacy in the setting of brain metastasis from breast cancer.

Methods: Our panel of breast cells was composed of four cell lines: T-47D (ER/PR+, Her2-, luminal A), MCF-7 (ER/PR+, Her2-, luminal A), MDA-MB-231 (TNBC, basal B), MDA-MB-468 (TNBC, basal A). The presence of cilengitide targets, $\beta 3$ and $\beta 5$ integrin, was first determined. Cell detachment was determined by cell counting, cell proliferation was determined by MTS proliferation assay, and apoptosis was measured by Annexin $V$ staining and flow cytometry. The efficacy of cilengitide treatment alone was analyzed, followed by assessment of combined cilengitide and radiation treatment. Integrin $\beta 3$ knockdown was performed, followed by cilengitide and radiation treatment to test for incomplete target inhibition by cilengitide, in high $\beta 3$ expressing cells.
\end{abstract}

Results: We observed that all cell lines examined expressed both $\beta 3$ and $\beta 5$ integrin and that cilengitide was able to induce cell detachment and reduced proliferation in our panel. Annexin $\mathrm{V}$ assays revealed that a portion of these effects was due to cilengitide-induced apoptosis. Combined treatment with cilengitide and radiation served to further reduce proliferation compared to either treatment alone. Following $\beta 3$ integrin knockdown, radiosensitization in combination with cilengitide was observed in a previously non-responsive cell line (MDA-MB-231). Clonogenic assays suggested little radiosensitization effects of cilengitide.

Conclusions: Cilengitide appears to enhance radiation response in preclinical models of breast cancer. These data suggest that the combination of radiation therapy and cilengitide may prove to be effective where radiation is utilized for the treatment of gross disease in breast cancer, such as in the setting of brain metastasis.

Keywords: Cilengitide, Breast cancer, Brain metastasis, Radiation

\footnotetext{
* Correspondence: TL@osumc.edu

${ }^{\dagger}$ Equal contributors

'Department of Radiation Oncology, The Ohio State University, Columbus, $\mathrm{OH} 43210$, USA

${ }^{2}$ Comprehensive Cancer Center, The Ohio State University, Columbus, OH 43210, USA

Full list of author information is available at the end of the article
} 


\section{Background}

Brain metastasis from breast cancer occurs in approximately $5 \%$ of patients overall, and in $10-16 \%$ of patients with metastatic disease [1]. Incidence is thought to be on the rise, as systemic therapy advances lead to better local tumor control and improved survival. Current treatments for these metastases include whole brain radiotherapy, surgery, stereotactic radiosurgery, and chemotherapy [2]. Outcomes for these patients are poor with median survivals ranging from 3.4-25.3 months based on the Graded Prognostic Assessment (GPA) [3]. Radiation is commonly used in the treatment of brain metastasis from breast cancer, where there can be gross disease present in the brain. Given that a significant percentage of these patients succumb to their metastatic disease and demonstrate local progression of their disease in the brain, we sought to investigate agents that may demonstrate additive or synergistic effects with radiation in the setting of breast cancer.

Integrins play a role in regulating cell-extracellular matrix interactions as well as cell signaling pathways that regulate adhesion, growth, motility, and survival [4]. Integrins are expressed on endothelial cells, and play an important role in angiogenesis [5], but have also been identified on a number of cancer cell types [6-8]. $\alpha \mathrm{V} \beta 3$ integrins specifically have been identified to play a direct role in tumor cell growth as well as invasion and metastasis [9,10]. $\alpha \mathrm{V} \beta 3$ integrins were shown to be critical for metastatic growth of breast cancer cells in the brain [11]. Therefore, we further examined targeting integrin signaling in combination with radiation.

Cilengitide is a cyclic RGD containing pentapeptide that targets $\alpha \mathrm{V} \beta 3$ and $\alpha \mathrm{V} \beta 5$ integrins [12]. This inhibitor has been shown to block glioma cell growth via cell detachment and induction of apoptosis in an in vitro model [13]. In vivo, cilengitide has been shown to inhibit metastatic bone colonization by the breast cancer cell line MDAMB-231 [14].

In the context of radiation therapy, combination treatment with cilengitide has been shown to radiosensitize lung cancer cell lines [15], with lung cancer representing the tumor site with the highest incidence of metastasis to the brain [1]. Combination therapy of cilengitide and radioimmunotherapy with an L6 antigen targeting antibody conjugated with the beta-emitter ${ }^{90} \mathrm{Y}$ has shown to improve outcomes of primary breast tumors in a xenograft model of breast cancer [16]. The combination of cilengitide and external beam radiotherapy has yet to be studied in the context of breast cancer.

We therefore set out to determine if a combination therapy of cilengitide and radiation could be of benefit for breast cancer brain metastases patients. To this end we tested the effect of cilengitide in combination with radiation in a panel of breast cancer cell lines, among them cell lines that have previously been used to study brain metastases from breast cancer.

\section{Methods}

\section{Cell culture and drug treatment}

T-47D (ER/PR+, Her2-, luminal A), MCF-7 (ER/PR+, Her2-, luminal A), MDA-MB-231 (TNBC, basal B), MDAMB-468 (TNBC, basal A) cell lines cells were purchased from ATCC. T-47D (RPMI-1640 medium supplemented with $10 \%$ fetal bovine serum, $1 \%$ penicillin/streptomycin, and 0.2 Units $/ \mathrm{ml}$ bovine insulin) and MCF-7 (Eagle's minimum essential medium supplemented with $10 \%$ fetal bovine serum, $1 \%$ penicillin/streptomycin, and $0.01 \mathrm{mg} / \mathrm{ml}$ bovine insulin) cell lines were maintained at $37^{\circ} \mathrm{C}$ in a 5\% $\mathrm{CO}_{2}$ atmosphere. MDA-MB-231 and MDA-MB-468 (Leibovitz's L-15 medium supplemented with $10 \%$ fetal bovine serum and $1 \%$ penicillin/streptomycin) cell lines were maintained at $37^{\circ} \mathrm{C}$ in an air atmosphere. Cilengitide was acquired through the Cancer Therapy Evaluation Program (CTEP) from Merck. Irradiation was performed using a RS2000 Biological Irradiator (Rad-Source) with $160 \mathrm{kV} \mathrm{x}$-rays with a $0.3 \mathrm{~mm}$ copper filter in place at a dose rate of approx. $1.2 \mathrm{~Gy} / \mathrm{min}$. All statistical comparisons were performed using repeated measures ANOVA with a Tukey's multiple comparison test. All research (non-human subject research, non-animal research) was done in accordance with institutional guidelines.

\section{Western blotting}

Untreated cells were washed and trypsinized, followed by pelleting and freezing at $-80^{\circ} \mathrm{C}$. Upon thawing cells pellets were resuspended in RIPA lysis buffer $(50 \mathrm{mM}$ Tris- $\mathrm{HCl}$, $\mathrm{pH}$ 8.0, with $150 \mathrm{mM}$ sodium chloride, $1.0 \%$ Igepal CA630 (NP-40), $0.5 \%$ sodium deoxycholate, and $0.1 \%$ sodium dodecyl sulfate) supplemented with the addition of Protease inhibitor cocktail (Sigma), phosphatase inhibitor cocktail 1 (Sigma), phosphatase inhibitor cocktail 2 (Sigma), and phenylmethylsulfonyl fluoride (Sigma). Protein concentration was determined with a BCA Protein Assay kit (Thermo Scientific) and 50ug was loaded per well in a 4-20\% TGE precast gel (Bio-rad), followed by transfer to PVDF membranes. Membranes were blocked using $5 \%$ bovine serum albumin in TBS-T, followed by overnight incubation at $4^{\circ} \mathrm{C}$ with primary antibody solution for $\beta 3$ or $\beta 5$ Integrin (1:1000, cell signaling). Blots were incubated with anti-rabbit secondary antibody (1:2000, cell signaling) was at RT for $1 \mathrm{hr}$. Blots were then developed using Immobilon Western Chemiluminescent HRP Substrate (Millipore) in the VersaDoc imaging system (Bio-rad).

\section{Flow cytometry - apoptosis}

Apoptosis assays were performed using the Alexa Fluor ${ }^{\circ}$ 488 Annexin V/Dead Cell Apoptosis Kit (Life Technologies) according to the manufacturer's instructions. Briefly, cells were plated and allowed to attach overnight. The following morning, cells were treated with indicated 
cilengitide doses and allowed to incubate for 48 hours. Apoptotic induction controls were treated with $16.6 \mathrm{uM}$ cisplatin 24 hours before staining. After 48 hours, cells were harvested (floating and attached cells) and stained according to manufacturer's instructions. Single stained and unstained controls were included. Flow cytometry was performed on a BD Facs LSR II flow cytometer under guidance from flow cytometry core staff. Samples were analyzed using Flow Jo software.

\section{Clonogenic assay}

All cell lines were trypsinized and counted, followed by dilution to appropriate levels. Cells were seeded in triplicate in $2 \mathrm{ml}$ growth media into 6-well tissue culture dishes and allowed to attach overnight. The following morning, the indicated doses of cilengitide were added to the cells. Irradiation was performed at the indicated doses 1 hour after drug treatment. Cells were then maintained at $37^{\circ} \mathrm{C}$ in the appropriate atmosphere for 10 days to 1 month until appropriate sized colonies had formed. Once colonies were visible, cells were stained in $0.5 \%$ crystal violet in methanol for 2 hours. Colonies were counted with the use of a dissecting microscope with a cutoff of 50 cells. Dose enhancement ratios are reported for $37 \%$ cell survival.

\section{Cell counting}

Cells were plated at $2 \times 10^{5}$ cells/well and allowed to attach. Cell counting was performed following $1 \mathrm{hr}$ treatment with the indicated cilengitide doses. Detached cells were removed and wells were washed with PBS. Remaining attached cells were then trypsinized and diluted 1:2 with trypan blue. Live cells were counted in duplicate using the Nexelcom Cellometer Auto T4 cell counter (Nexelcom Bioscience). Remaining cell viability was at least $95 \%$.

\section{MTS assay}

Proliferation assays were performed using the CellTiter Aqueous One Solution Cell Proliferation Assay (Promega) according to modified manufacturer's instructions. $2 \times 10^{4}$ cells/well were plated in $500 \mathrm{ml}$ growth medium in triplicate. Cells were treated at the indicated dose of cilengitide on day 0 . Control readings were measured on day 0 to control for plating inaccuracies and cell metabolism differences. The CellTiter solution was diluted 1:6 and $240 \mathrm{ul} /$ well was added. Plates were incubated for $3 \mathrm{hrs}$ at $37^{\circ} \mathrm{C}$, followed by removal of $100 \mathrm{ul}$ of MTS solution. Absorbance at $490 \mathrm{~nm}$ was obtained using a Mithras LB 940 plate reader (Berthold Technologies). Treated cells were left for 96 hours and absorbencies were obtained as above. ITGB3 knockdowns were performed using ON-TARGET Plus SMARTpool Human ITGB3 siRNA and Control NonTargeting siRNA. Cells were treated with $25 \mathrm{nM}$ siRNA for 48 hours before plating. Day 4 values were controlled to Day 0 values within each cell line. Values were also controlled to untreated cell values and reported as \% Cell Viability compared to untreated cells. For the ITGB3 knockdown experiments, normalization was performed against the untreated control cells for each group (Non-Targeting cells or ITGB3 knockdown cells respectively).

\section{Results}

Cilengitide target expression in breast cell lines

$\beta 3$ and $\beta 5$ integrins, found as dimers with $\alpha \mathrm{V}$ integrins, are the targets for the inhibitor cilengitide. $\alpha \mathrm{V} \beta 3$ integrin is thought to represent the primary target, while $\alpha \mathrm{V} \beta 5$ is an alternate target. We examined the breast cancer cell lines T-47D, MCF-7, MDA-MB-231, and MDA-MB-468 for $\beta 3$ and $\beta 5$ integrin expression by western blot. As shown in Figure 1 , we detected both $\beta 3$ and $\beta 5$ integrin in all of our cell lines ( $\beta 3$ integrin can be detected as possibly 3 different bands of 97,110 , and $130 \mathrm{kDa}$ ). T-47D and MCF-7 cell lines expressed $\beta 3$ integrin at low levels, while MDA-MB231 and MDA-MB-468 cell lines expressed $\beta 3$ integrin at higher levels than the other two cell lines studied. $\beta 5$ integrin was most strongly expressed in T-47D cells, at intermediate levels in MCF-7 and MDA-MB-231 cells, and only very lowly expressed in MDA-MB-468 cells.

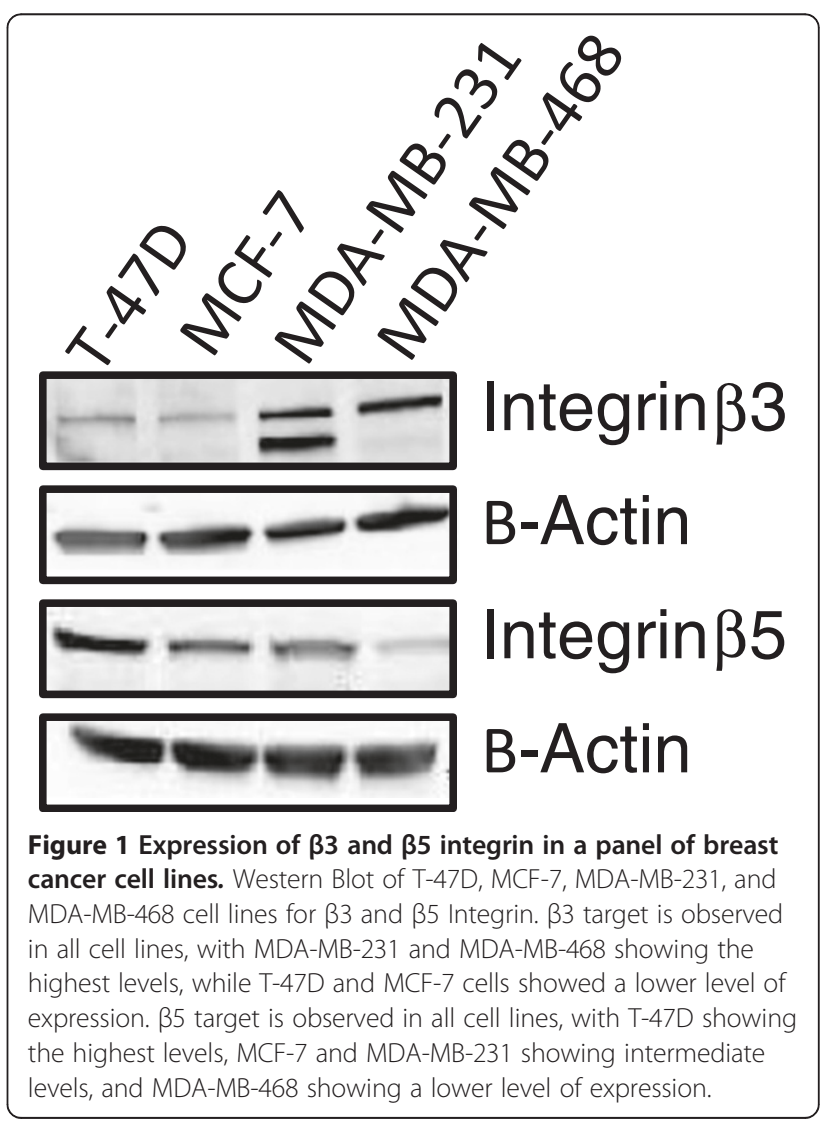




\section{Effects of cilengitide alone Cell detachment}

Once expression of the target of cilengitide was verified, we assessed the effects of cilengitide treatment as a single therapy in our panel of cell lines. Previous studies have shown that cilengitide causes cell detachment in culture $[13,15]$. The role of integrins in cell adhesion to uncoated plastic dishes is probably related to serum vitronectin adhering to the plastic [17]. We wanted to examine this cellular detachment in our panel of cell lines. Briefly cells were treated with cilengitide ( $500 \mathrm{nM}, 5 \mathrm{uM}$, and $20 \mathrm{uM}$ ) for 1 hour. Detached cells were then washed away with PBS, and remaining attached cells were trypsinized and counted. We observed that in most of our cell lines there was a dose dependent effect of cilengitide on cell detachment. At the highest dose tested $(20 \mathrm{uM}) \mathrm{T}-47 \mathrm{D}$ cells showed the most marked response, with almost complete detachment of cells following 1 hour of treatment (Figure 2). MDA-MB468 cells showed little to no cell detachment at this early timepoint, even at this highest dose. Both of the other cell lines tested showed a moderate response to the $20 \mathrm{uM}$ dose, losing about $50 \%$ of plated cells (Figure 2). Most of these cells, however, are likely to be merely detached, as opposed to dead. Cells treated for 1 hour with $20 \mathrm{uM}$ cilengitide to cause detachment, were then washed with PBS and re-plated into a new cell culture dish. At least a part of these washed cells were able to attach and grow normally (data not shown). Cells left in cilengitide up to 96 hours remained detached (data not shown).

\section{Growth inhibition}

The same trends discussed above were observed in our panel of cell lines in a longer term proliferation assay. Breast cancer cell lines were plated, allowed to attach overnight, and then treated the following morning with varying doses of cilengitide $(1 \mathrm{uM}, 5 \mathrm{uM}, 10 \mathrm{uM}$, and $20 \mathrm{uM}$ ). The cells were then allowed to grow for 96 hours in the presence of cilengitide and cell viability was determined. As shown in Figure 3 the cell responses at 96 hours following cilengitide treatment are similar to the early 1 hour detachment response. In this assay, T-47D cells still showed the most marked response, with almost complete cell loss compared to untreated cells at the 20 uM dose (Figure 3). MDA-MB-468 cells again showed no response to cilengitide treatment, even at the $20 \mathrm{uM}$ dose. In this assay, MDA-MB-231 cells had a reduced
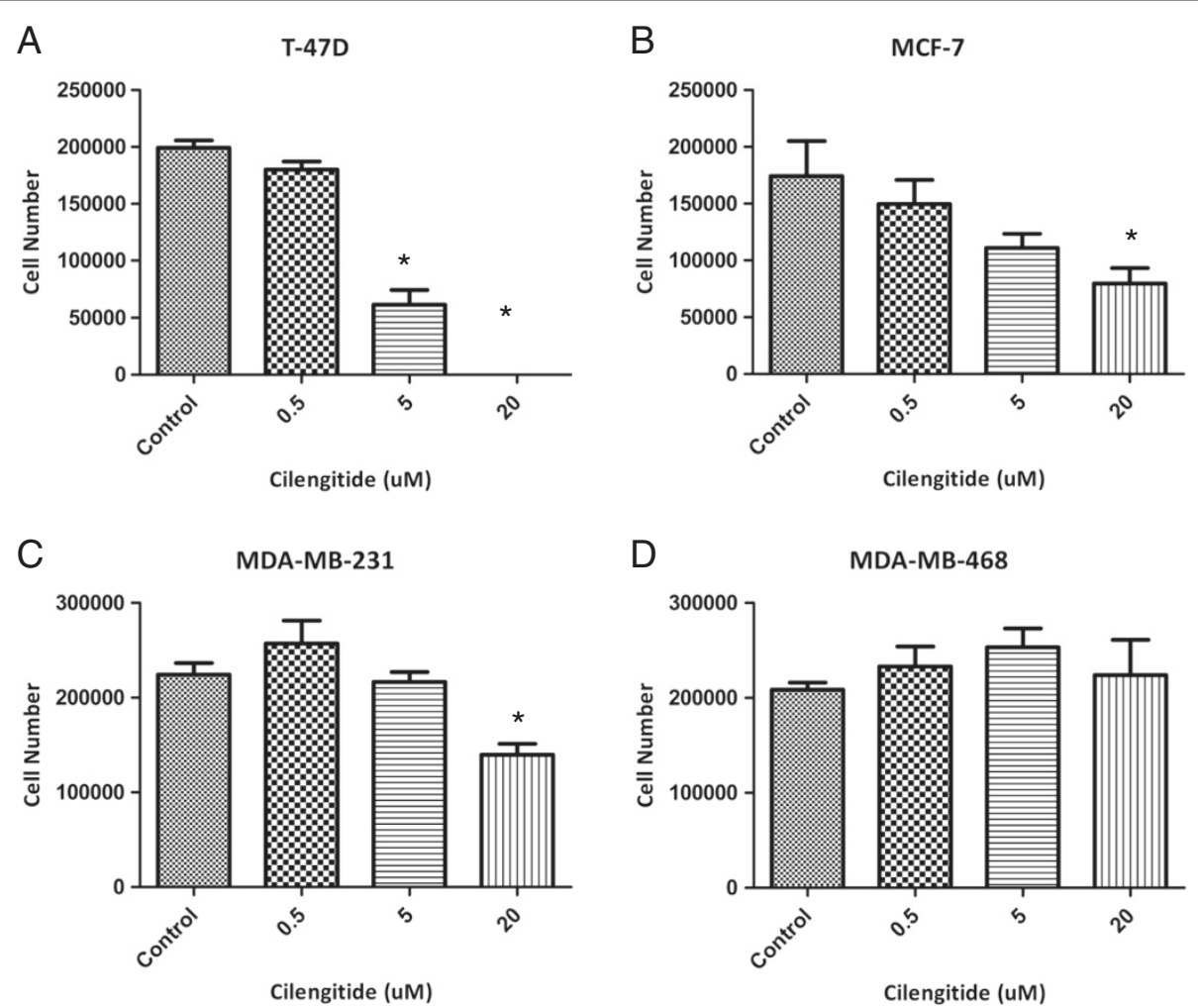

Figure 2 Cell detachment following 1 hr Cilengitide treatment. Cells were treated with cilengitide for 1 hr, washed with PBS, and remaining attached cells were trypsinized and counted. A) T-47D cells showed a strong dose response, with almost complete cell detachment at 20 uM cilengitide treatment. B) MCF-7 cells showed moderate cell detachment similar to C) MDA-MB-231 cells. D) MDA-MB-468 cells showed little to no cell detachment following this short exposure to cilengitide. Figures show Mean \pm SEM and represent the average of three experiments. $*=0.05$. 

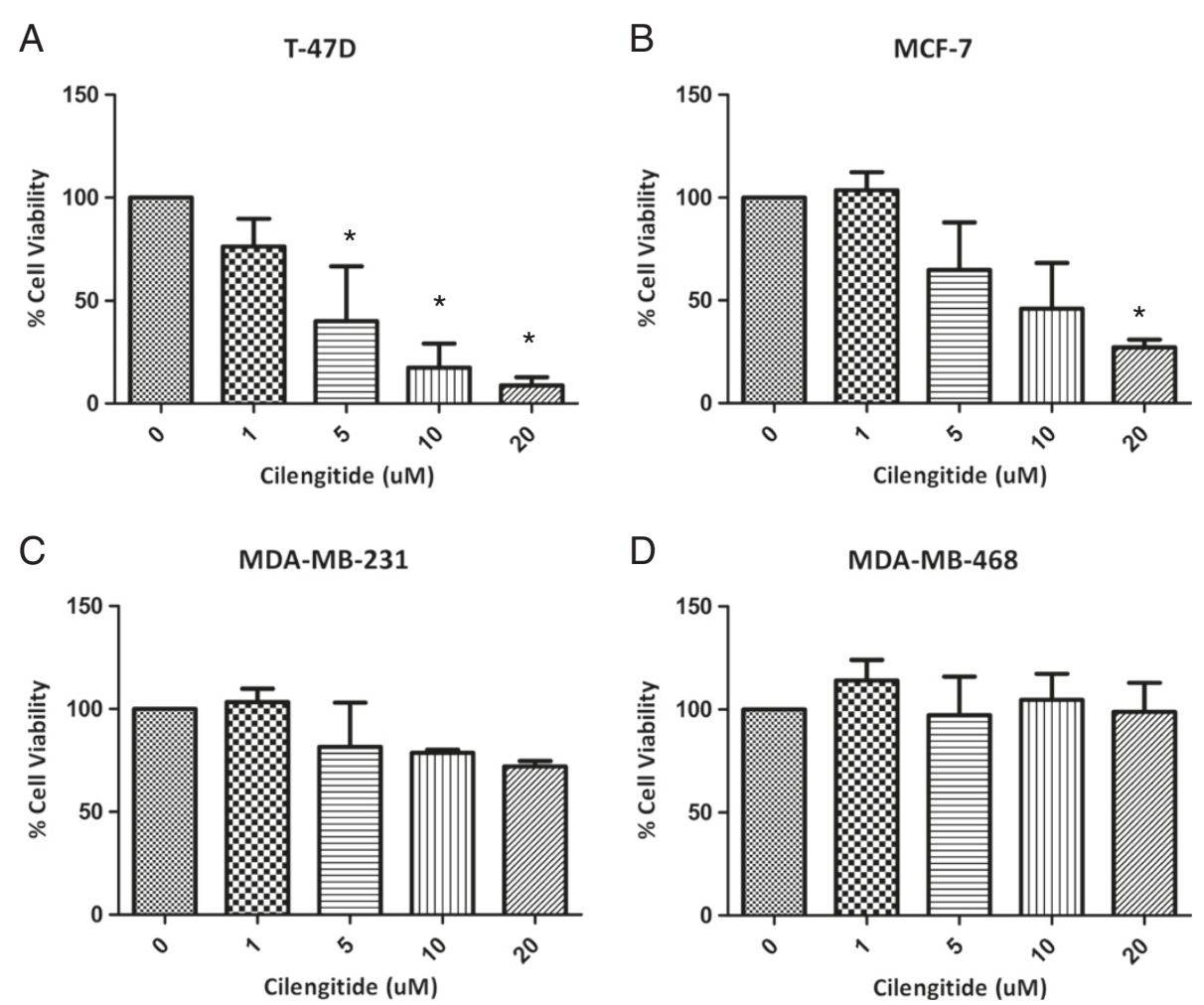

Figure 3 MTS proliferation assay following prolonged cilengitide treatment. Cells were treated with Cilengitide for 96 hours, followed by washing and addition of an MTS substrate. After 4 hour incubation, absorbance was read and cell viability as percent of control treated cells was reported. A) T-47D cells show the most marked response, with little to no cell growth at the 20 uM cilengitide dose. B) MCF-7 cells show a moderate growth delay. C) MDA-MB-231 cells also show a moderate growth delay. D) MDA-MB-468 cells show little to no growth delay compared to controls at this 96 hour timepoint. Most cell lines showed a dose response to cilengitide (A-C) with only MDA-MB-468 cells showing no effects. Figures show Mean \pm SEM and represent the average of three experiments. ${ }^{*}=\leq 0.05$.

response when compared to the previous early cell detachment assay, showing only $25 \%$ reduction in cell proliferation after 96 hours of cilengitide exposure compared to controls as opposed to $50 \%$ of cells detached after 1 hour (Figures $2 \mathrm{C}$ and 3C). On the other hand MCF7 cells showed a more noticeable response, with nearly an $80 \%$ reduction in cell proliferation compared to the $50 \%$ of cells lost after $1 \mathrm{hr}$ of treatment (Figures 2B and 3B). Overall these results are consistent with the early cell detachment results. Cilengitide appears to have a significant effect, on both cell attachment and cell growth, in our breast cancer cell lines.

\section{Cilengitide induces apoptosis}

To examine the mechanism by which cilengitide reduces viability in our cells, we performed an apoptosis assay at an intermediate timepoint (48 hours) following cilengitide treatment. First, cilengitide treatment alone induced apoptosis in all cell lines except for the MDA-MB-468 cells, and this apoptotic induction was dose dependent (Figure 4). This pattern of cell line dependent cilengitide responsiveness is consistent with our earlier cell detachment results (Figure 2) and cell proliferation data (Figure 3). In this assay, we again observed that the most significant effects of cilengitide occurred in the T-47D cell line. The late apoptotic/dead (Q2) and early apoptotic (Q3) events were increased from Q2 - 6.67\%, Q3 - 7.69\% in T-47D control cells to Q2 - 19.0\%, Q3 - 23.9\% in cells treated with $20 \mathrm{uM}$ of cilengitide. MCF-7 cells showed the next highest response (control; Q2 - 2.25\%, Q3 - 5.49\% to $20 \mathrm{uM}$; Q2 17.7\%, Q3 - 23.4\%). MDA-MB-231 (control; Q2 - 1.67\%, Q3 - 6.36\% to $20 \mathrm{uM}$; Q2 - 3.39\%, Q3 - 13.6\%) showed a more moderate effect of cilengitide treatment in this assay. MDA-MB-468 cells showed no response to cilengitide, as in the other assays performed (control; Q2 - 2.43\%, Q3 $2.10 \%$ to $20 \mathrm{uM}$ : Q2 - 2.85\%, Q3 - 3.79\%). Our findings indicate that cilengitide is able to induce apoptosis and death in some of our tested breast cancer cell lines when used as a single treatment. This finding is in line with other reports which indicate that cells detached by cilengitide treatment go on to apoptose [13]. However, even in a cell line/ cilengitide dose combination (T-47D and $20 \mathrm{uM}$ ) where $100 \%$ of cells appear detached microscopically after one hour (data not shown, compare to Figure 1) there are still approximately $44 \%$ of live cells (Quadrant 4 ) at $48 \mathrm{hrs}$. It was also observed in T-47D cells exposed to cilengitide for 96 hours that following washing cells were able to attach 


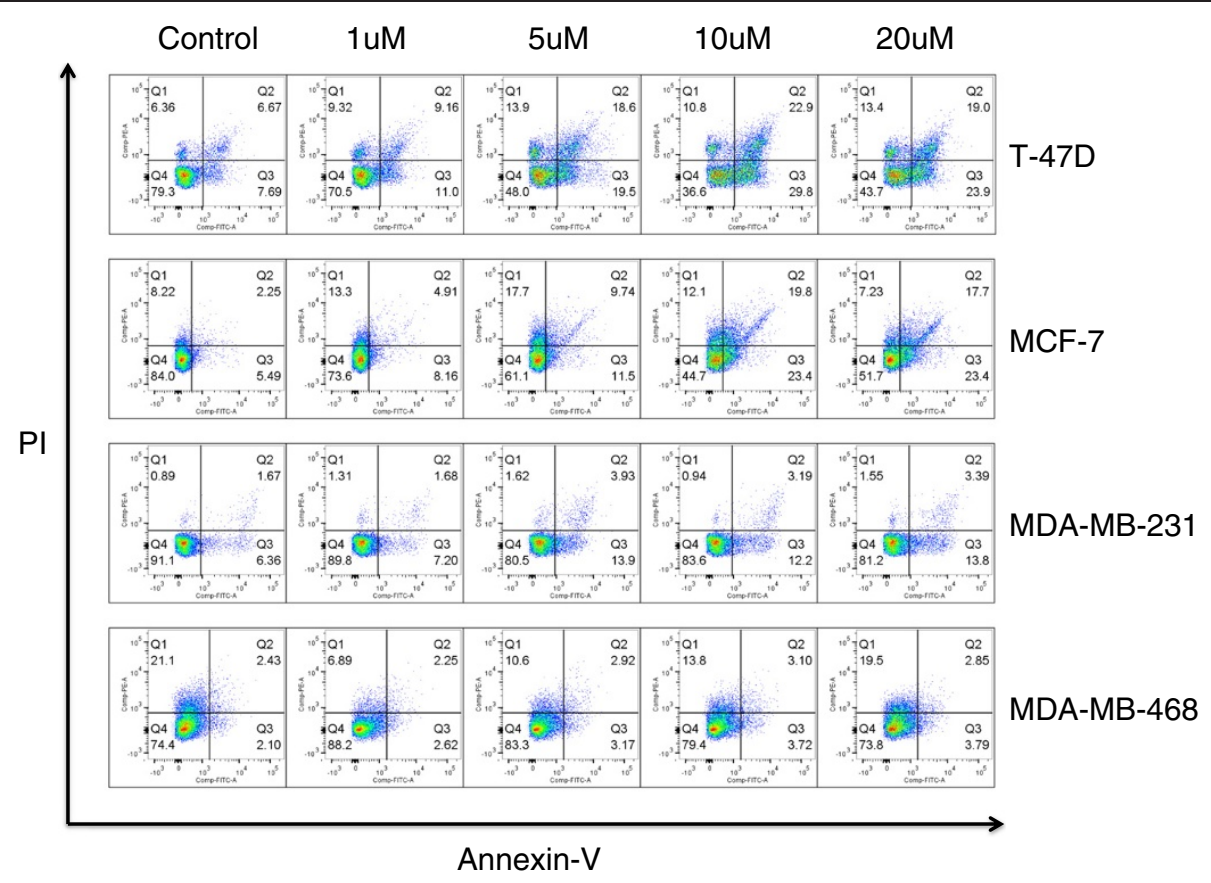

Figure 4 Cilengitide induces apoptosis in breast cell lines. Cells were treated for 48 hours with the reported cilengitide doses and then harvested for Annexin V/PI staining and analysis by flow cytometry. T-47D cells showed the highest level of apoptotic/dead cells. MCF-7 cells showed the next highest levels of apoptotic/dead cells, with levels very similar to T-47D cells. MDA-MB-231 cells show a moderate induction of apoptosis. MDA-MB-468 cells show no apoptotic induction, which mirrors the cell detachment and proliferation assay results. Experiment was performed three times with similar results. Figures are from a representative experiment.

and grow (data not shown) indicating that even at 96 hours, not all of the detached cells are dead or dying.

\section{Effects of cilengitide in combination with radiation}

After characterizing the response of our cell lines to cilengitide as a single therapy, we sought out to determine if cilengitide could serve as a radiosensitizer. First, we performed a proliferation assay (MTS) as discussed earlier (Figure 3) with ionizing radiation (IR) added to the cilengitide treatment groups. T-47D, MCF-7, and MDAMB-231 cells were treated at 4 Gy, while MDA-MB-468 cells were treated at $2 \mathrm{~Gy}$. An initial test had shown that MDA-MB-468 cells were especially sensitive in terms of viability at 96 hours after IR with 4 Gy (data not shown). All of the cell lines appeared to have reduced viability after combination treatment of cilengitide and IR compared to IR alone (Figure 5). For T-47D and MCF-7 cells those differences were statistically significant, while for MDAMB-231 and MDA-MB-468 we observed only a trend towards improved efficacy of the combination treatment. Clonogenic assays showed only very minor increases in radiosensitivity following cilengitide treatment in some of the cell lines tested (Figure 6). These results indicate that cilengitide is enhancing the effects of radiation in our panel of cells, and that there may be an additive effect for this combination treatment.
It was observed that the least responsive cell lines in this study were those with the highest levels of $\beta 3$ and lowest levels of $\beta 5$ integrin target. The association of high expression levels of integrin $\beta 3$ and poor response to cilengitide in our small cell line panel suggests that integrin $\beta 3$ is the relevant cilengitide target. One possibility for these results is that this target is not completely inhibited at the cilengitide doses used. Since integrin $\beta 5$ expression was associated with responsive cell lines, we determined that this target was likely being adequately inhibited by the cilengitide doses studied. To examine if these cells were unresponsive to cilengitide treatment due to incomplete $\beta 3$ blockade at tested treatment doses, $\beta 3$ integrin was knocked down in the MDA-MB-231 cell line. Approximately $50 \%$ knockdown was achieved in these cell lines (Figure 7A). 48 hours following knockdown, cells were plated and treated as in the above experiments. Figure 7B shows that cells with reduced $\beta 3$ integrin show enhanced response to radiation and cilengitide combination treatment, while previously they were minimally responsive to cilengitide and radiation treatment (Figure 5).

\section{Discussion}

Brain metastases from breast cancer occur in 10-16\% of patients who eventually develop metastatic disease [1] The advent of brain metastases often is a life altering event for breast cancer patients and not uncommonly impacts 

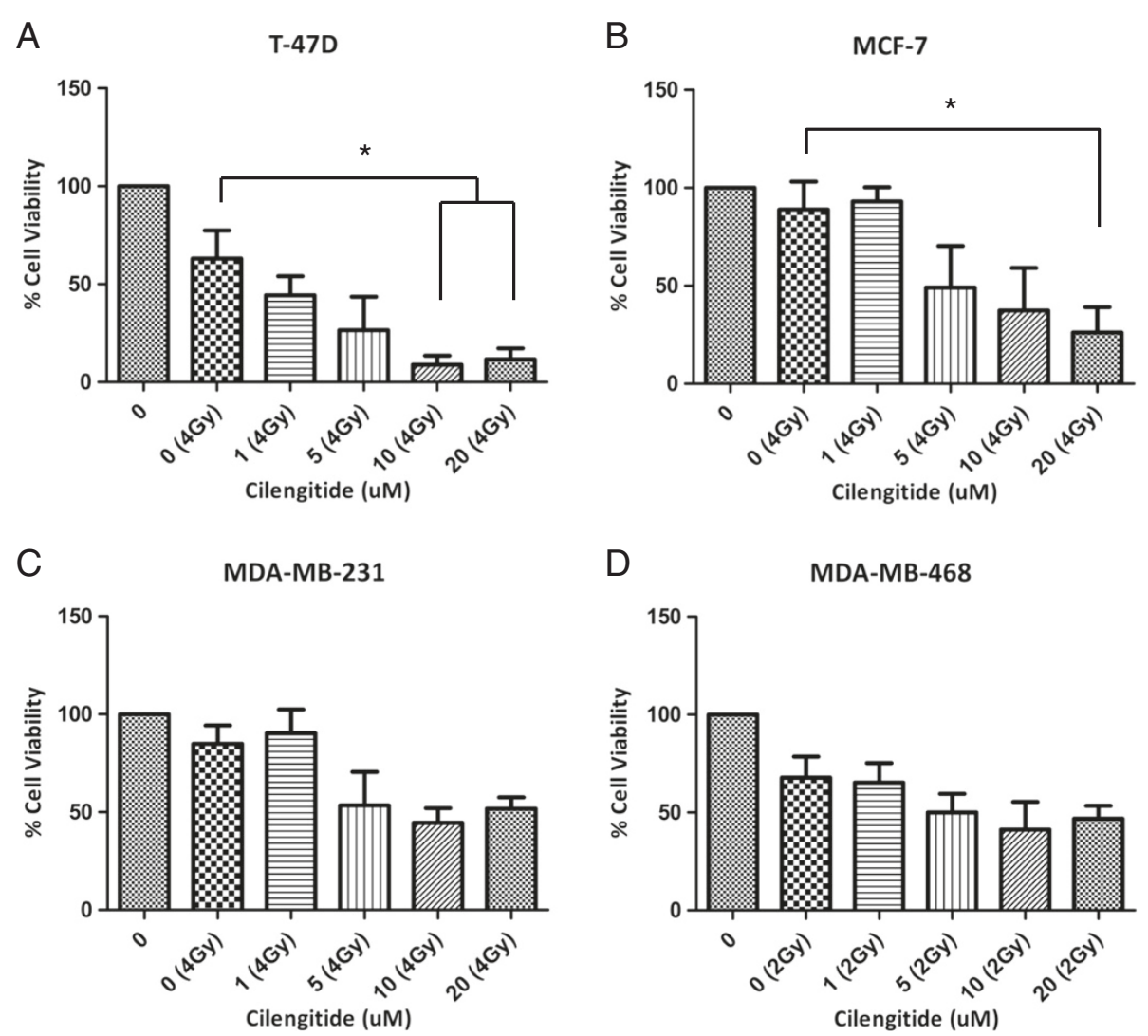

Figure 5 MTS proliferation assay following prolonged cilengitide treatment in combination with ionizing radiation. Cells were treated in the same manner as in Figure 3, with the addition that for the radiation groups, cells were treated with the indicated dose of IR following $1 \mathrm{hr}$ of cilengitide treatment. All four cell lines show a combination effect where cilengitide in combination with radiation has an enhanced growth delay effect when compared to IR alone treated cells. A) T-47D cells show this combination effect at lower doses of cilengitide than other cells lines. B) MCF-7 cells show an improvement over radiation alone with cilengitide combination. C) MDA-MB-231 cells show a trend toward an enhanced effect of cilengitide in combination with IR treatment. D) MDA-MB-468 cells also show only a trend toward increased growth delay in the combination groups versus the radiation alone group. Figures show Mean \pm SEM and represent the average of three experiments. ${ }^{*}=\leq 0.05$.

quality of life and cognitive functioning. Outcomes for patients with brain metastases are poor with median survival times ranging from 3.4-25.3 months depending on GPA [3]. There is an urgent need for novel therapeutic approaches to improve outcomes in this patient population.

In this study we present our findings suggesting cilengitide treatment can induce cellular detachment and apoptosis, and reduce proliferation in a panel of breast cancer cell lines. While this is, to our knowledge, the first report to describe cilengitide effects in combination with radiation in a panel of breast cancer cell lines our findings are consistent with previous reports describing induction of apoptosis, reduced proliferation, and cellular detachment after cilengitide treatment in glioma [13] and lung cancer [15] models.

The primary target of cilengitide is $\alpha v \beta 3$ integrin. Integrin expression on tumor cells plays a role in activating many of the major cell survival pathways including ERK and PI3K signaling [18]. These pathways are known to lead to increased tumor cell proliferation, migration, and resistance to apoptosis [19]. Furthermore inhibition of those pathways can result in radiosensitizing effects in many cancer cell lines [20]. Therefore, we speculate that these pathways could mediate the treatment effects in breast cancer cell lines as well, and it is not surprising that the integrin inhibitor cilengitide has anti-tumor activity in lung and glioma models or in breast cancer models as here presented.

The magnitude of cilengitide effects on breast cancer cell lines, however, was fairly heterogeneous among the cell lines tested. Given that only four cell lines were tested no definitive conclusions can be drawn at this time as to which breast cancer brain metastases patient subpopulations might derive the most benefit from cilengitide containing treatment regimens. It appeared that cell lines that are ER/PR positive and Her2 negative or cell lines with lower expression levels of integrin $\beta 3$ had the most significant response to cilengitide treatment in this study. Experimentally reducing the expression of integrin $\beta 3$ in a cell line with high integrin $\beta 3$ expression (MDA-MB-231) resulted in increased efficacy of cilengitide and radiation. Given that integrin $\beta 3$ knockdown can sensitize cells to the tested cilengitide doses, 

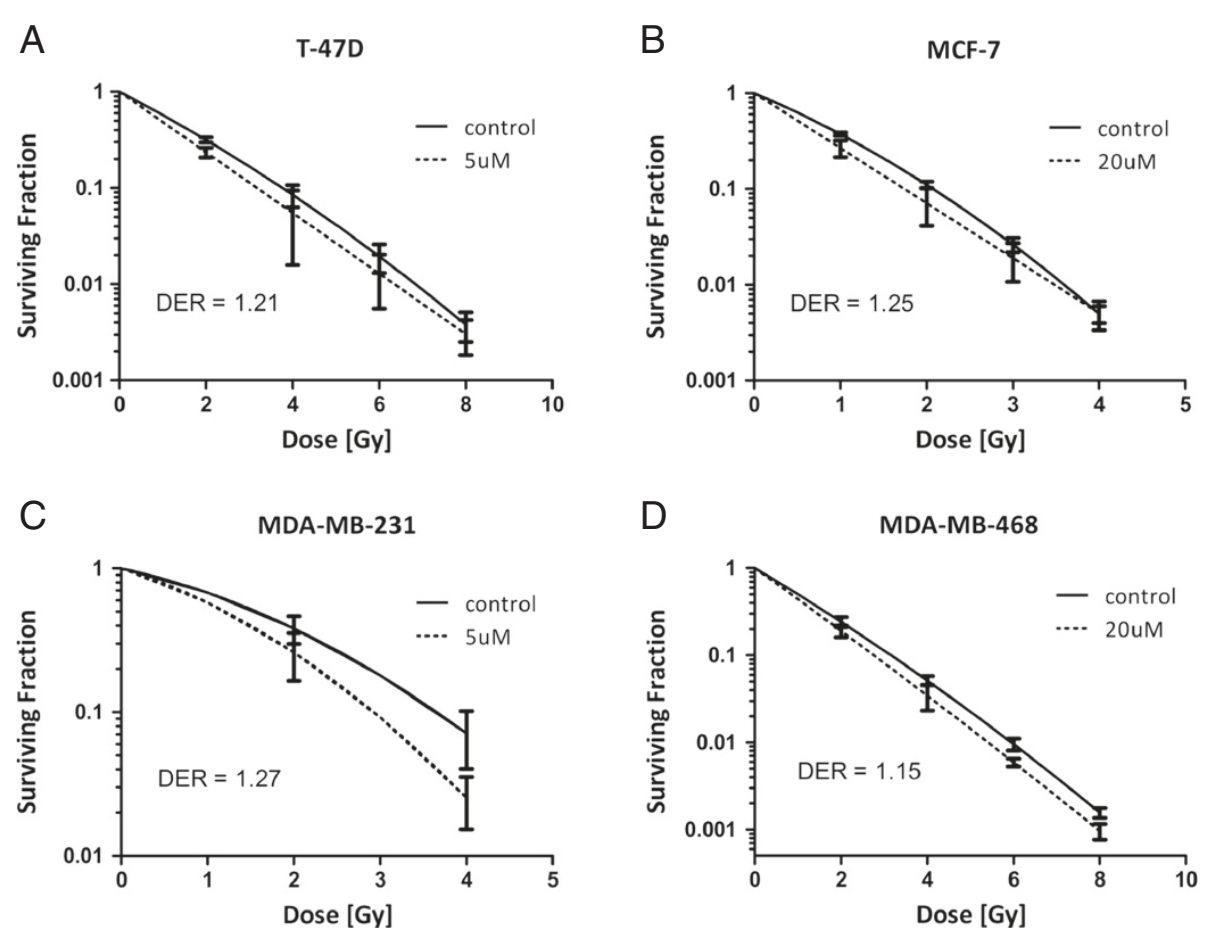

Figure 6 Clonogenic assay in combination with cilengitide in breast cell lines. Clonogenic assays were performed with our panel of breast cell lines. Cells were plated at specific cell numbers and treated with indicated doses of cilengitide. After 1 hr of treatment, cells were irradiated and then incubated for up to 4 weeks to allow colony formation. Cells were then stained and counted. All four cell lines show small dose enhancement ratios with the addition of cilengitide, indicating no or very little radiosensitization effects. A) T-47D (DER - 1.21), B) MCF-7 (DER - 1.25), C) MDA-MB231 (DER - 1.27), D) MDA-MB-231 (DER - 1.15). Figures represent the average of three experiments.

A

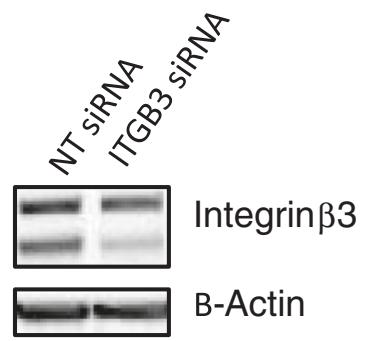

MDAMB-231
B

MDA-MB-231

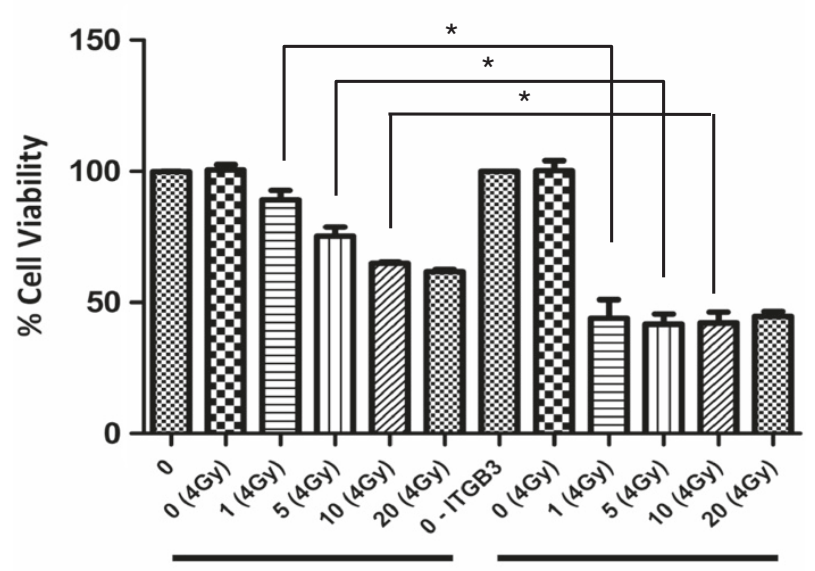

NT SiRNA
ITGB3 siRNA

\section{Cilengitide (uM)}

Figure 7 MTS proliferation assay following ITGB3 knockdown in MDA-MB-231 cells. Cells were treated with 25nM siRNA (either NT or ITGB3) for 48 hours before being treated in the same manner as described in Figure 5. A) Target knockdown was confirmed via western blot. B) Cells treated with NT siRNA showed a similar pattern to previous results, while cells treated with ITGB3 siRNA showed a pronounced radiosensitization in combination with cilengitide treatment. NT untreated controls and ITGB3 untreated controls were used for normalization within their respective groups. The ratio of ITGB3 knockdown/NT treated cell viability cell viability at 96 hours is 0.935 . Figures show Mean \pm SEM and represent the average of three experiments. ${ }^{*}=\leq 0.05$. 
it appears that this target is not completely inhibited in cell lines with high expression of integrin $\beta 3$. While it is still possible that integrin $\beta 5$ inhibition is responsible for the enhanced response seen in the T-47D or MCF-7 cell lines, these results indicate that at least a portion of the cilengitide resistance in our other cell lines is due to the high levels of integrin $\beta 3$ expression observed. While the results of our proliferation assays have shown clear statistical benefit of combination therapy compared to either therapy alone in some breast cell lines, our clonogenic data shows little to no formal radiosensitization effect. Our results indicate a more significant benefit of combined radiation and cilengitide treatment at earlier timepoints compared to the long term results of the clonogenic assays. This difference could well be due to the different timepoints tested. However, the performed normalization of the radiated arm to the cilengitide alone treated cells likely contributes to this observed difference as well. Our data however is similar to reports using glioma models [21] where modest radiosensitizing effects of cilengitide were found. Previously it has been reported that cilengitide and radiation have additive effects in glioma cell lines [22]. Our data indicate a similar additive nature of combination cilengitide and radiation therapy in breast cancer cell lines, rather than a synergistic or supra-additive effect. These results are more limited in scope when compared to more significant radiosensitizing effects of cilengitide reported in NSCLC models [15]. The underlying reasons for the differences in potency of cilengitide as a radiosensitizer in different cancers remain to be identified and is subject to further studies. Despite the differences in efficacy seen comparing our assays, combined cilengitide and radiation treatment appeared to be beneficial for most of the tested breast cancer cell lines.

In summary, our data suggest cilengitide treatment has anti-tumor activity in breast cancer cell line models, and that combination treatment with radiation appears to affect cells more than either treatment alone. Given that some of the cell lines used in our study have previously been used as breast cancer brain metastases models [23], and that cilengitide potentially can achieve therapeutically relevant concentrations in the brain, we conclude that combined cilengitide and radiation treatment could be a promising therapeutic strategy for a subset of breast cancer brain metastases patients.

\section{Conclusions}

Combined cilengitide and radiation therapy appears to be more efficacious than either treatment alone in breast cancer cell lines. This study, along with previous work highlights the promise of cilengitide therapy in combination with radiation. Based on the ability of cilengitide to accumulate in the brain combination treatment of cilengitide and radiation could be beneficial clinically for a subset of breast cancer brain metastases patients.

\begin{abstract}
Abbreviations
GPA: Graded prognostic assessment; CTEP: Cancer therapy evaluation Program; IR: Ionizing radiation.
\end{abstract}

\section{Competing interests}

The authors declare that they have no competing interests.

\section{Authors' contributions}

$T L$ conceived of the study, and participated in its design and coordination and helped to draft the manuscript. JP participated in the design of the study, participated in the Western blot analysis and performed apoptosis, proliferation, and clonogenic survival assays, and helped draft the manuscript. DP conceived of the study, and revised the manuscript critically for intellectual content. BL participated in the cell culture studies, and participated in drafting the manuscript. Al participated in Western blot analysis and participated in revising the manuscript. AH participated in the design of the study, participated in statistical analysis, and participated in drafting the manuscript. WM participated in the design of the study, and participated in drafting the manuscript. JW participated in designing of the study, and revised the manuscript critically for intellectual content. AC conceived of the study, and participated in coordination of the study, and revised the manuscript critically for intellectual content. All authors read and approved the final manuscript.

\section{Acknowledgement}

Cilengitide was acquired through the Cancer Therapy Evaluation Program (CTEP) from Merck.

The work was supported by awards from the James Comprehensive Cancer Center (to AC)

The project described was supported by Award Number Grant 8UL1TR000090-05 from the National Center For Advancing Translational Sciences. The content is solely the responsibility of the authors and does not necessarily represent the official views of the National Center For Advancing Translational Sciences or the National Institutes of Health.

\section{Author details}

${ }^{1}$ Department of Radiation Oncology, The Ohio State University, Columbus, $\mathrm{OH}$ 43210, USA. ${ }^{2}$ Comprehensive Cancer Center, The Ohio State University, Columbus, OH 43210, USA. ${ }^{3}$ Burkhardt Brain Tumor and Neuro-Oncology Center, Cleveland Clinic, Cleveland, OH 44195, USA. ${ }^{4}$ Pharmaceutical Industries Division, National Research Center, Cairo, Dokki 12622, Egypt.

Received: 6 August 2013 Accepted: 18 October 2013

Published: 23 October 2013

\section{References}

1. Barnholtz-Sloan JS, Sloan AE, Davis FG, Vigneau FD, Lai P, Sawaya RE: Incidence proportions of brain metastases in patients diagnosed (1973 to 2001) in the Metropolitan Detroit Cancer Surveillance System. J Clin Oncol 2004, 22:2865-2872.

2. Sharma M, Abraham J: CNS metastasis in primary breast cancer. Expert Rev Anticancer Ther 2007, 7:1561-1566.

3. Sperduto PW, Kased N, Roberge D, Xu Z, Shanley R, Luo X, Sneed PK, Chao ST, Weil RJ, Suh J, Bhatt A, Jensen AW, Brown PD, Shih HA, Kirkpatrick J, Gaspar LE, Fiveash JB, Chiang V, Knisely JP, Sperduto CM, Lin N, Mehta M: Summary report on the graded prognostic assessment: an accurate and facile diagnosis-specific tool to estimate survival for patients with brain metastases. J Clin Oncol 2012, 30:419-425.

4. Desgrosellier JS, Cheresh DA: Integrins in cancer: biological implications and therapeutic opportunities. Nat Rev Cancer 2010, 10:9-22.

5. Silva R, D'Amico G, Hodivala-Dilke KM, Reynolds LE: Integrins: the keys to unlocking angiogenesis. Arterioscler Thromb Vasc Biol 2008, 28:1703-1713.

6. Sloan EK, Pouliot N, Stanley KL, Chia J, Moseley JM, Hards DK, Anderson RL: Tumor-specific expression of alphavbeta3 integrin promotes spontaneous metastasis of breast cancer to bone. Breast Cancer Res 2006, 8:R20.

7. Bello L, Francolini M, Marthyn P, Zhang J, Carroll RS, Nikas DC, Strasser JF, Villani R, Cheresh DA, Black PM: Alpha(v)beta3 and alpha(v)beta5 integrin expression in glioma periphery. Neurosurgeny 2001, 49:380-389. discussion 390.

8. Adachi M, Taki T, Higashiyama M, Kohno N, Inufusa H, Miyake M: Significance of integrin alpha5 gene expression as a prognostic factor in node-negative non-small cell lung cancer. Clin Cancer Res 2000, 6:96-101. 
9. Ria R, Vacca A, Ribatti D, Di Raimondo F, Merchionne F, Dammacco F: Alpha (v)beta(3) integrin engagement enhances cell invasiveness in human multiple myeloma. Haematologica 2002, 87:836-845.

10. Cruet-Hennequart S, Maubant S, Luis J, Gauduchon P, Staedel C, Dedhar S: alpha(v) integrins regulate cell proliferation through integrin-linked kinase (ILK) in ovarian cancer cells. Oncogene 2003, 22:1688-1702.

11. Lorger M, Krueger JS, O'Neal M, Staflin K, Felding-Habermann B: Activation of tumor cell integrin alphavbeta3 controls angiogenesis and metastatic growth in the brain. Proc Natl Acad Sci U S A 2009, 106:10666-10671.

12. Mas-Moruno $\mathrm{C}$, Rechenmacher F, Kessler H: Cilengitide: the first antiangiogenic small molecule drug candidate design, synthesis and clinical evaluation. Anticancer Agents Med Chem 2010, 10:753-768.

13. Oliveira-Ferrer L, Hauschild J, Fiedler W, Bokemeyer C, Nippgen J, Celik I, Schuch G: Cilengitide induces cellular detachment and apoptosis in endothelial and glioma cells mediated by inhibition of FAK/src/AKT pathway. J Exp Clin Cancer Res 2008, 27:86-9966-27-86.

14. Bretschi M, Merz M, Komljenovic D, Berger MR, Semmler W, Bauerle T: Cilengitide inhibits metastatic bone colonization in a nude rat model. Oncol Rep 2011, 26:843-851.

15. Albert JM, Cao C, Geng L, Leavitt L, Hallahan DE, Lu B: Integrin alpha v beta 3 antagonist Cilengitide enhances efficacy of radiotherapy in endothelial cell and non-small-cell lung cancer models. Int I Radiat Oncol Biol Phys 2006, 65:1536-1543.

16. Burke PA, DeNardo SJ, Miers LA, Lamborn KR, Matzku S, DeNardo GL: Cilengitide targeting of alpha(v)beta(3) integrin receptor synergizes with radioimmunotherapy to increase efficacy and apoptosis in breast cancer xenografts. Cancer Res 2002, 62:4263-4272.

17. Steele JG, Dalton BA, Underwood PA, Smith GJ: Differences in adhesion to tissue culture plastic of clonally related transformed and control sublines from an epithelial cell strain. J Cell Sci 1991, 100(Pt 1):195-203.

18. Keely PJ: Mechanisms by which the extracellular matrix and integrin signaling act to regulate the switch between tumor suppression and tumor promotion. J Mammary Gland Biol Neoplasia 2011, 16:205-219.

19. Mendoza MC, Er EE, Blenis J: The Ras-ERK and PI3K-mTOR pathways: cross-talk and compensation. Trends Biochem Sci 2011, 36:320-328.

20. McKenna WG, Muschel RJ, Gupta AK, Hahn SM, Bernhard EJ: The RAS signal transduction pathway and its role in radiation sensitivity. Oncogene 2003, 22:5866-5875

21. Mikkelsen T, Brodie C, Finniss S, Berens ME, Rennert JL, Nelson K, Lemke N, Brown SL, Hahn D, Neuteboom B, Goodman SL: Radiation sensitization of glioblastoma by cilengitide has unanticipated schedule-dependency. Int J Cancer 2009, 124:2719-2727.

22. Maurer GD, Tritschler I, Adams B, Tabatabai G, Wick W, Stupp R, Weller M: Cilengitide modulates attachment and viability of human glioma cells, but not sensitivity to irradiation or temozolomide in vitro. Neuro Oncol 2009, 11:747-756.

23. Palmieri D, Smith QR, Lockman PR, Bronder J, Gril B, Chambers AF, Weil RJ, Steeg PS: Brain metastases of breast cancer. Breast Dis 2006, 26:139-147.

doi:10.1186/1748-717X-8-246

Cite this article as: Lautenschlaeger et al:: In vitro study of combined cilengitide and radiation treatment in breast cancer cell lines. Radiation Oncology 2013 8:246.

\section{Submit your next manuscript to BioMed Central and take full advantage of:}

- Convenient online submission

- Thorough peer review

- No space constraints or color figure charges

- Immediate publication on acceptance

- Inclusion in PubMed, CAS, Scopus and Google Scholar

- Research which is freely available for redistribution

Submit your manuscript at www.biomedcentral.com/submit
Biomed Central 